\title{
Risk stratification of patients with nonalcoholic fatty liver disease using a case identification pathway in primary care: a cross-sectional study
}

\author{
Abdel Aziz Shaheen MBBCh MPH, Kiarash Riazi MBBCh, Alexandra Medellin MD, Deepak Bhayana MD, \\ Gilaad G. Kaplan MD MPH, Jason Jiang MSc, Roy Park MD, Wendy Schaufert RN, Kelly W. Burak MD MSc, \\ Monica Sargious MD, Mark G. Swain MD MSc
}

\section{Abstract}

Background: Identification of patients with nonalcoholic fatty liver disease (NAFLD) with advanced liver fibrosis in primary care remains an unmet need. Our primary objective was to implement a pathway driven by shear wave elastography (SWE) to facilitate risk stratification of patients with NAFLD within primary care and evaluate whether SWE assessment can reduce referrals of patients with NAFLD at low risk for fibrosis to hepatology.

Methods: A multidisciplinary NAFLD clinical care pathway was codeveloped by hepatologists, radiologists and primary care physicians in Calgary to provide access to SWE-based screening of patients with NAFLD risk factors in primary care. The study outcome measures were estimated NAFLD-related referrals to the hepatology service in Calgary after implementation of the NAFLD pathway and characteristics of patients with NAFLD at risk for advanced fibrosis. The NAFLD pathway was implemented in January 2018 and was made available to all primary care physicians in the Calgary Health Zone. Patients with NAFLD who had liver stiffness (SWE value $\geq 8.0 \mathrm{kPa}$ ) or an inconclusive assessment were referred to hepatology. A serum liver fibrosis score was also measured with the fibrosis-4 (FIB-4) index, and performance of an FIB-4 index score of 1.30 or greater to risk stratify patients with NAFLD was evaluated. Demographic, clinical and laboratory characteristics of study groups were compared.

Results: Between March and October 2018, 2084 patients with suspected NAFLD were evaluated. Nonalcoholic fatty liver disease was confirmed by ultrasonography in 1958 (94.1\%). A majority of the cohort had elevated liver enzyme values (1028 [52.5\%]) and obesity (body mass index $\geq 30)(1063 / 1764$ [60.3\%]). Most patients with NAFLD (1791 [91.5\%]) had an SWE value less than $8.0 \mathrm{kPa}$ and were not referred to hepatology. Sixty-seven patients (3.4\%) had an SWE value of $8.0 \mathrm{kPa}$ or more, and 100 (5.1\%) had an inconclusive SWE; these patients were referred to hepatology. Using an FIB-4 index score cut-off of 1.30 would have led to hepatology referral of 396/1251 patients (31.6\%).

Interpretation: Implementation of a primary care-accessible SWE pathway for patients with NAFLD facilitated fibrosis risk stratification and greatly reduced hepatology referrals. Using the FIB-4 index score alone would led to higher rates of referral of patients with NAFLD.

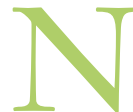

onalcoholic fatty liver disease (NAFLD) is common in patients with diabetes, obesity, dyslipidemia and metabolic syndrome, and has become the leading cause of liver disease in North America. ${ }^{1,2}$ Patients with NAFLD at risk for advanced fibrosis require specialist care for urgent assessment and management of liver cirrhosis complications. ${ }^{3,4}$ Intense lifestyle modification and treatment of underlying risk factors are the main approach for treating patients with NAFLD with advanced fibrosis. ${ }^{3,5}$ Early clinical intervention and patient education lead to better outcomes, including possible reversal of histopathologic liver changes, especially in patients who achieve a $7 \%-10 \%$ weight loss. ${ }^{6,7}$ Therefore, recent guidelines have emphasized the high yield of tar- geting patients with NAFLD who have advanced fibrosis for intense lifestyle modification. . $^{3,5}$

Although awareness of NAFLD among primary care physicians is increasing, identifying patients with NAFLD

Competing interests: Abdel Aziz Shaheen and Mark Swain received an Investigator-Sponsored Research grant from Gilead Sciences. No other competing interests were declared.

This article has been peer reviewed.

Correspondence to: Abdel Aziz Shaheen, aashahee@ucalgary.ca CMAJ Open 2020. DOI:10.9778/cmajo.20200009 
at high risk for advanced fibrosis in primary care remains challenging. ${ }^{8-10}$ Furthermore, a strategy to help primary care physicians better triage patients with NAFLD for specialist care based on liver fibrosis is lacking. ${ }^{11}$

Noninvasive serum liver fibrosis scores, such as the fibrosis-4 (FIB-4) index, and ultrasonography-based modalities including transient elastography and shear wave elastography (SWE) have been used to assess liver fibrosis severity in patients with NAFLD. ${ }^{12-14}$ Transient elastography and SWE are valid and reliable measures of liver fibrosis. ${ }^{15,16}$ Our primary objective was to implement an SWE-driven pathway to facilitate risk stratification of patients with NAFLD within primary care and evaluate whether SWE assessment can reduce referrals of patients with NAFLD at low risk for fibrosis to hepatology.

\section{Methods}

\section{Background and setting}

Owing to the growing prevalence of NAFLD, there has been a substantial increase in referrals from primary care physicians for NAFLD assessment to the hepatology service in Calgary, Alberta, leading to increasing wait times. The hepatology service has a single-entry-point central access and triage process for 10 hepatologists and covers a large urban population of about 1.4 million. Specifically, patients with NAFLD represented about $40 \%$ (monthly median 510/1235) of referrals to the hepatology service from January 2016 to December 2017. Most patients with NAFLD assessed by the hepatology service were found to be at low risk for liver fibrosis and did not require further follow-up.

\section{Development of clinical care pathway}

Calgary hepatology leadership (M.G.S., head of the gastroenterology division in the Calgary Health Zone) proposed developing a Calgary NAFLD care pathway to the leadership of Calgary's Primary Care Networks. Details on collaboration among care providers to develop the Calgary NAFLD clinical care pathway (CN-CCP) and outreach to primary care physicians can be found in Appendix 1 (available at www.cmajopen.ca/content/8/2/E370/suppl/DC1).

The $\mathrm{CN}-\mathrm{CCP}$ is accessible to primary care physicians for patients with risk factors for NAFLD, including diabetes, increased body mass index, dyslipidemia or metabolic syndrome, previous imaging evidence of fatty liver and elevated liver enzyme levels. Patients with other chronic liver diseases, including heavy alcohol consumption (> 2 standard alcohol drinks per day for men, $>1$ for women), viral hepatitis B or C, and immune-mediated liver disorders, are excluded.

In the pathway, patients with probable NAFLD and no exclusion criteria are referred directly by primary care physicians to community-based radiology providers for SWE assessment. Patients found to be at risk for advanced fibrosis (SWE value $\geq 8.0 \mathrm{kPa}$ or inconclusive $\mathrm{SWE}$ assessment) are recommended for hepatology referral through a single citywide central referral access point. A cut-off of $8 \mathrm{kPa}$ or greater was chosen because the negative predictive value of advanced fibrosis for values less than $8 \mathrm{kPa}$ is more than $96 \% .^{13,17}$ Patients at low risk (SWE value $<8.0 \mathrm{kPa}$ ) are managed within primary care with a standardized management plan that includes lifestyle modifications.

For long-term patient follow-up, it was decided by all leadership groups that a reminder fax should be sent by the radiology provider to the SWE-ordering physician, suggesting repeat SWE 3 years after the initial SWE. In addition, primary care physicians can contact hepatologists for advice through a dedicated advice telephone line as needed.

The CN-CCP (Appendix 2, available at www.cmajopen. $\mathrm{ca} /$ content/8/2/E370/suppl/DC1) was formally implemented in January 2018. It was made available to all primary care physicians in the Calgary Health Zone and is published online (www.specialistlink.ca/files/NAFLD_Pathway_May2019.pdf).

\section{Design, participants and data source}

Adult patients in Calgary with a confirmed NAFLD diagnosis during SWE assessment between March and November 2018 were included in this cross-sectional study. Our manuscript is compliant with Revised Standards for Quality Improvement Reporting Excellence (SQUIRE-2). ${ }^{18}$

The CN-CCP database includes all adult Calgary residents with suspected NAFLD referred by primary care physicians for SWE as of January 2017. The database contains patient demographic characteristics; comorbidities; laboratory data (complete blood count, lipid profile [triglycerides, cholesterol, highdensity lipoprotein and low-density lipoprotein], hemoglobin $\mathrm{A}_{\mathrm{lc}}\left[\mathrm{HbA}_{\mathrm{lc}}\right]$ concentration, international normalized ratio and liver enzyme values [alanine aminotransferase (ALT), aspartate aminotransferase (AST), alkaline phosphatase, albumin, $\gamma$-glutamyl transpeptidase and creatinine]), medications for diabetes, dyslipidemia and hypertension; and SWE findings (assessment date, reliable study [yes/no], median and interquartile range of speed [in metres/second] based on 10 measurements, median elasticity [in kilopascals], portal vein diameter, liver echogenicity and attenuation, and liver contour and echotexture). Completeness of the CN-CCP demographic and radiologic data elements is $95 \%$; data sources are updated monthly. The validity of the CN-CCP is continually evaluated by comparing its elements to Alberta Health Services databases.

\section{Covariates}

We included the following variables from the $\mathrm{CN}-\mathrm{CCP}$ database: age, sex, body mass index, type 2 diabetes mellitus (defined as $\mathrm{HbA}_{\mathrm{lc}}$ concentration $>6.4 \%$, a diagnosis code of type 2 diabetes by a physician or use of type 2 diabetes treatment), impaired fasting glucose (defined as $\mathrm{HbA}_{\mathrm{cc}}$ concentration $5.7 \%-6.4 \%$ ), hypertension (defined by a physician diagnosis code or drug treatment for hypertension) and laboratory investigations.

Laboratory data were obtained at baseline (within $3 \mathrm{mo}$ of SWE). Patients were considered to have elevated liver enzyme values if ALT values were at or above the upper limit of normal ( $\geq 30 \mathrm{U} / \mathrm{L}$ for men, $\geq 25 \mathrm{U} / \mathrm{L}$ for women). ${ }^{19}$ As AST was often not routinely ordered by primary care physicians, we described the patient characteristics of those with available AST values and FIB-4 index 
scores compared to patients with missing values (AST is required for calculation of FIB-4 index score).

\section{Liver stiffness assessment}

Shear wave elastography is a real-time ultrasonography-based technique widely used to assess liver stiffness (i.e., fibrosis) using a pulse induced by acoustic radiation force through tissue to create shear waves. ${ }^{20,21}$ It was performed with a 2-dimensional Canon Aplio i800 ultrasonography system. A standard abdominal ultrasonography examination (with the patient fasting) was performed by a trained technologist (completed $\geq 100$ supervised SWE examinations) or a subspecialty body radiologist with SWE experience. ${ }^{21,22}$ If the examiner was unable to obtain a technically reliable shear wave (using interquartile range $[\mathrm{IQR}] /$ median $\leq 15 \%$ for metres/second or $\leq 30 \%$ for kilopascals), ${ }^{22}$ the SWE assessment was deemed inconclusive.

\section{Case confirmation}

All patients evaluated with the $\mathrm{CN}-\mathrm{CCP}$ received abdominal ultrasonography and SWE. Patients with homogeneous liver echotexture, normal echogenicity and contour, and no signal attenuation were identified as not having fatty liver. Patients with liver steatosis were considered to have NAFLD. ${ }^{20,22}$ Patients with failed SWE (inability to obtain a valid SWE assessment) were excluded.

\section{Outcomes}

Our primary outcome measure was NAFLD-related referral to the hepatology service in Calgary after implementation of the $\mathrm{CN}-\mathrm{CCP}$. The secondary outcome measure was characteristics of patients with NAFLD at risk for advanced fibrosis.

\section{Statistical analysis}

We described patient characteristics according to SWE results. We used the $\chi^{2}$ test and the Wilcoxon rank-sum test or Kruskal-Wallis test to examine demographic, laboratory and clinical differences between patients with and without fibrosis at baseline according to SWE values. Logistic regression models identified independent predictors of an SWE value of $8.0 \mathrm{kPa}$ or greater, or inconclusive SWE measurements.

Logistic regression models were adjusted for age, sex, body mass index, ALT at baseline and comorbidities. We reported all regression model estimates as adjusted odds ratio (OR) with accompanying $95 \%$ confidence interval (CI).

Further analyses evaluated possible differences between patients with complete laboratory investigations (for calculating FIB-4 index score) and those with incomplete laboratory investigations. Among patients with an available FIB-4 index score, we assessed the performance of a score of 1.30 or greater as an index test for risk stratification of patients with NAFLD compared to an SWE value of $8.0 \mathrm{kPa}$ or greater. An FIB-4 index score cut-off value of 1.30 has shown high negative predictive value for advanced fibrosis $(>90 \%) .{ }^{13} \mathrm{We}$ assessed the rates of triggering a recommendation for hepatology referral with 7 SWE cut-off points between $6.0 \mathrm{kPa}$ and $9.0 \mathrm{kPa}$.
We performed all analyses using Stata/IC version 15.1 (StataCorp).

\section{Ethics approval}

The study protocol was approved by the Conjoint Health Research Ethics Board at the University of Calgary.

\section{Results}

A total of 2084 patients with suspected NAFLD were evaluated with the CN-CCP between March and November 2018 (Figure 1). Ultrasonography showed NAFLD in 1958 patients (94.1\%). The diagnosis of NAFLD varied by body mass index ( $\geq 30$ : 1223/1259 [97.1\%]; 25-29: 426/462 [92.2\%], $p<0.001$ ). Baseline clinical and laboratory investigations are presented in Table 1 . There was a slightly higher proportion of female patients (1052 [53.7\%]) with NAFLD; the median age was 55 (IQR 45-63) years. The median SWE value was $4.4 \mathrm{kPa}$ (IQR $3.7-5.5 \mathrm{kPa}$ ), and the median FIB-4 index score was 0.99 (IQR $0.69-1.48)$.

\section{Patient classification by shear wave elastography result}

Three patients had failed SWE and were excluded from analysis. Of the 1958 patients with NAFLD, 1791 (91.5\%) had an SWE value less than $8.0 \mathrm{kPa}$ and were not referred to

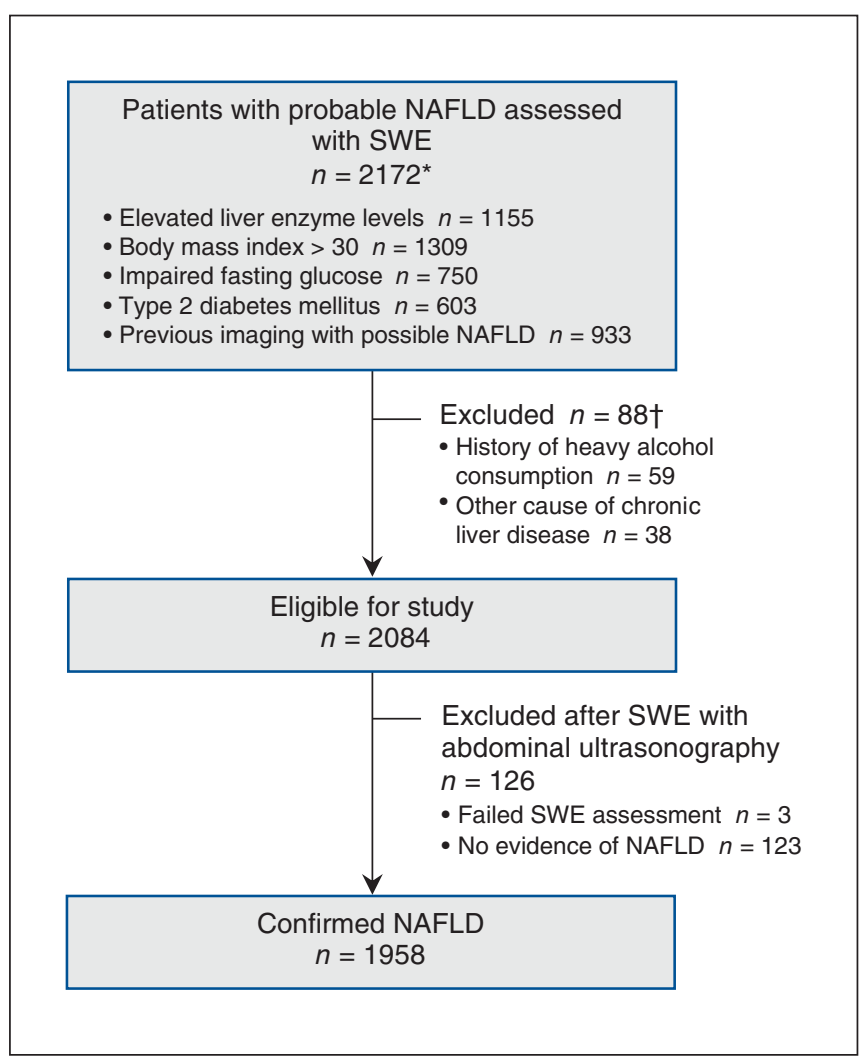

Figure 1: Flow chart showing selection of study cohort. Note: NAFLD = nonalcoholic fatty liver disease, SWE = shear wave elastography. *Some patients had more than 1 condition. †Some patients had both exclusion criteria. 
Table 1: Characteristics of patients with nonalcoholic fatty liver disease according to shear wave elastography result

\begin{tabular}{|c|c|c|c|c|c|}
\hline \multirow[b]{2}{*}{ Characteristic } & \multicolumn{4}{|c|}{ Group; no. (\%) of patients* } & \multirow[b]{2}{*}{$p$ value } \\
\hline & $\begin{array}{l}\text { Total cohort } \\
n=1958\end{array}$ & $\begin{array}{l}\text { SWE value } \\
\begin{array}{l}\geq 8 \mathrm{kPa} \\
n=67\end{array}\end{array}$ & $\begin{array}{l}\text { Inconclusive } \\
\text { result } \\
n=100\end{array}$ & $\begin{array}{l}\text { SWE value } \\
\quad<8 \mathrm{kPa} \\
n=1791\end{array}$ & \\
\hline Age, yr, median (IQR) & $55(45-63)$ & $61(49-69)$ & $57(47-65)$ & $54(45-63)$ & 0.004 \\
\hline Female sex & $1052(53.7)$ & $44(65.7)$ & $55(55.0)$ & $953(53.2)$ & 0.1 \\
\hline $\begin{array}{l}\text { Body mass index, median (IQR) } \\
(n=1764)\end{array}$ & $32.0(28.0-36.6)$ & $37.2(31.7-40.8)$ & $40.6(33.4-47.2)$ & $31.6(27.8-35.9)$ & $<0.001$ \\
\hline \multicolumn{6}{|l|}{$\begin{array}{l}\text { Baseline laboratory investigations, } \\
\text { median (IQR) }\end{array}$} \\
\hline ALT, U/L $(n=1944)$ & $38(25-60)$ & $37(21-63)$ & $31(20-52)$ & $38(25-61)$ & 0.05 \\
\hline AST, U/L $(n=1273)$ & $29(21-42)$ & $36(23-56)$ & $27(19-50)$ & $29(21-41)$ & 0.06 \\
\hline Albumin, $g / L(n=1473)$ & $39(37-41)$ & $37(36-39)$ & $37(34-39)$ & $39(37-41)$ & $<0.001$ \\
\hline ALP, U/L $(n=1667)$ & $77(64-94)$ & $91(68-107)$ & $85(71-118)$ & $76(63-93)$ & $<0.001$ \\
\hline GGT, U/L $(n=1627)$ & $45(27-87)$ & $87(36-133)$ & $48(29-138)$ & $45(27-82)$ & $<0.001$ \\
\hline INR $(n=875)$ & $1.0(1.0-1.1)$ & $1.0(1.0-1.1)$ & $1.0(1.0-1.2)$ & $1.0(1.0-1.0)$ & $<0.001$ \\
\hline Platelet count, $10 \times 9 / \mathrm{L}(n=1922)$ & 250 (209-294) & $214(169-255)$ & $234(179-295)$ & $252(211-295)$ & $<0.001$ \\
\hline Triglycerides, $\mathrm{mmol} / \mathrm{L}(n=1830)$ & $1.80(1.28-2.61)$ & $1.79(1.23-2.37)$ & $1.71(1.28-2.30)$ & $1.81(1.28-2.64)$ & 0.2 \\
\hline Cholesterol, $\mathrm{mmol} / \mathrm{L}(n=1831)$ & $4.75(4.01-5.48)$ & $4.04(3.44-4.70)$ & $4.01(3.42-4.87)$ & $4.81(4.10-5.53)$ & $<0.001$ \\
\hline $\mathrm{HDL}, \mathrm{mmol} / \mathrm{L}(n=1831)$ & $1.16(0.95-1.39)$ & $1.11(0.90-1.34)$ & $1.10(0.92-1.31)$ & $1.16(0.96-1.39)$ & 0.09 \\
\hline LDL, mmol/L $(n=1831)$ & $2.61(1.95-3.27)$ & $2.07(1.57-2.55)$ & $2.10(1.62-2.71)$ & $2.66(2.03-3.33)$ & $<0.001$ \\
\hline Creatinine, $\mathrm{mmol} / \mathrm{L}(n=1895)$ & $74(60-87)$ & $67(51-79)$ & $66(54-79)$ & $74(61-88)$ & $<0.001$ \\
\hline Hemoglobin $\mathrm{A}_{10}, \%(n=1810)$ & $5.7(5.5-6.2)$ & $6.2(5.6-7.2)$ & $6.1(5.6-7.1)$ & $5.7(5.4-6.2)$ & $<0.001$ \\
\hline Diabetes mellitus & $562(28.7)$ & $38(56.7)$ & $50(50.0)$ & $474(26.5)$ & $<0.001$ \\
\hline Impaired fasting glucose & $657(33.6)$ & $12(17.9)$ & $24(24.0)$ & $621(34.7)$ & 0.002 \\
\hline Hypertension & $798(40.8)$ & $41(61.2)$ & $63(63.0)$ & $694(38.7)$ & $<0.001$ \\
\hline $\begin{array}{l}\text { Fibrosis-4 index score, median (IQR) } \\
(n=1251)\end{array}$ & $0.99(0.69-1.48)$ & $1.71(1.03-2.63)$ & $1.19(0.77-2.05)$ & $0.96(0.68-1.41)$ & $<0.001$ \\
\hline $\begin{array}{l}\text { Note: } A L P=\text { alkaline phosphatase, } A L T=\text { alanin } \\
\text { INR = international normalized ratio, IQR }=\text { inte } \\
{ }^{*} \text { Except where noted otherwise. } \\
\text { †For comparison between cohort subgroups }(3\end{array}$ & $\begin{array}{l}\text { inotransferase, } A S \\
\text { tile range, } L D L=10 \\
\text { ps). }\end{array}$ & $\begin{array}{l}\text { tate aminotransfera } \\
\text { lipoprotein, SWE }\end{array}$ & $\begin{array}{l}=\gamma \text {-glutamyl trans } \\
\text { vave elastography. }\end{array}$ & se, $\mathrm{HDL}=$ high-de & oprotein, \\
\hline
\end{tabular}

hepatology. Sixty-seven patients (3.4\%) had an SWE value of $8.0 \mathrm{kPa}$ or greater, and $100(5.1 \%)$ had inconclusive results; hepatology referral was recommended for these patients.

Compared to nonreferred patients, patients who were referred to hepatology were older (median age $54 \mathrm{yr}$ v. $61 \mathrm{yr}$ for those with $\mathrm{SWE}$ value $\geq 8.0 \mathrm{kPa}$ and $57 \mathrm{yr}$ for those with inconclusive results, $p<0.01$ ), had a higher body mass index (median 31.6 v. 37.2 and 40.6, respectively, $p<0.01$ ) and were more likely to have impaired fasting glucose or diabetes $(50$ [74.6\%] v. 1095 [61.1\%] and 74 [74.0\%], respectively, $p<0.01)$. Referred patients had similar ALT and AST levels as nonreferred patients. However, compared to nonreferred patients, referred patients had lower albumin levels (median $39 \mathrm{~g} / \mathrm{L} \mathrm{v}$. $37 \mathrm{~g} / \mathrm{L}$ for both those with SWE value $\geq 8.0 \mathrm{kPa}$ and those with inconclusive results, $p<0.001$ ), higher $\gamma$-glutamyl transpeptidase levels (median $45 \mathrm{U} / \mathrm{L}$ v. $87 \mathrm{U} / \mathrm{L}$ and $48 \mathrm{U} / \mathrm{L}$, respectively, $p<0.001$ ) and lower platelet counts (median $252 \times 10^{9} / \mathrm{L} \mathrm{v}$. $214 \times 10^{9} / \mathrm{L}$ and $234 \times 10^{9} / \mathrm{L}$, respectively, $\left.p<0.001\right)($ Table 1$)$.

\section{Stratification based on different shear wave elastography cut-off values}

Using SWE cut-off points between 6.0 and $9.0 \mathrm{kPa}$ led to different rates for triggering a hepatology referral recommendation. Specifically, 67 patients with NAFLD (3.4\%) had an SWE result of $8.0 \mathrm{kPa}$ or greater, $106(5.4 \%)$ had a result of $7.0 \mathrm{kPa}$ or greater, and $44(2.2 \%)$ had a result of $9.0 \mathrm{kPa}$ or greater (Appendix 3, Supplemental Table S1, available at www.cmajopen.ca/content/8/2/E370/suppl/DC1).

\section{Risk stratification using fibrosis-4 index score}

Patient characteristics did not differ between those with available FIB-4 index scores $(n=1251$ [63.9\%]) and those without ( $n=707$ [36.1\%]) (Appendix 3, Supplemental Table S2). With an FIB-4 index score cut-off of less than $1.30,855$ patients $(68.3 \%)$ would have been classified as being at low risk for advanced liver fibrosis, but 396 (31.6\%) would have been referred to hepatology (Table 2). However, 


\begin{tabular}{|c|c|c|}
\hline \multirow[b]{2}{*}{ SWE value, $\mathrm{kPa}$} & \multicolumn{2}{|c|}{ FIB-4 index score; no. (\%) of patients } \\
\hline & $\begin{array}{c}\geq 1.30 \\
n=396\end{array}$ & $\begin{array}{c}<1.30 \\
n=855\end{array}$ \\
\hline$\geq 8.0$ & $34(8.6)$ & $21(2.5)$ \\
\hline$<8.0$ & 362 (91.4) & 834 (97.5) \\
\hline
\end{tabular}

of patients referred to hepatology with an FIB-4 index score of 1.30 or higher, only $34(8.6 \%)$ had an SWE value of $8.0 \mathrm{kPa}$ or greater. Of the 855 patients with an FIB-4 index score less than 1.30, $21(2.5 \%)$ had an SWE value of $8.0 \mathrm{kPa}$ or greater. Therefore, agreement was observed in $69.4 \%$ of patients (868/1251). Using a higher FIB-4 index score cutoff of 2.24 would have led to hepatology referral of 128 patients $(10.2 \%)$.

\section{Predictors of need for hepatology referral}

In models adjusted for patient characteristics (including age, sex, obesity [body mass index $\geq 30$ ], type 2 diabetes, impaired fasting glucose, hypertension, and elevated ALT or AST level at baseline), obesity (adjusted OR 1.93, 95\% CI 1.01-3.75), type 2 diabetes (adjusted OR 2.22, 95\% CI 1.13-4.36) and hypertension (adjusted OR 2.18, 95\% CI 1.19-3.98) were the only independent predictors of an SWE value of $8.0 \mathrm{kPa}$ or greater (Table 3). Independent predictors of the need for hepatology referral were similar to those in the model predicting an $\mathrm{SWE}$ value of $8.0 \mathrm{kPa}$ or greater: patients with obesity had a threefold higher risk of needing a hepatology referral (adjusted OR 2.94, 95\% CI 1.85-4.69), and patients with type 2 diabetes and hypertension had a twofold higher risk (adjusted OR 2.33, 95\% CI 1.50-3.63, and adjusted OR $2.38,95 \%$ CI 1.62-3.50, respectively) (Table 3 ).

\section{Interpretation}

We report findings from a large North American primary care-based cohort of patients with NAFLD assessed for elevated liver stiffness values with a primary care-based NAFLD clinical pathway implementing SWE to evaluate liver fibrosis risk. We used this pathway to differentiate patients at low risk for advanced fibrosis, who do not require hepatology referral, from those who are at risk and could potentially benefit from hepatology referral. Overall, $3.4 \%$ of patients with NAFLD in our cohort had an SWE value of $8.0 \mathrm{kPa}$ or greater, suggesting they were at risk for advanced liver fibrosis, and 5.1\% had an inconclusive SWE assessment.

Similar to previous studies, ${ }^{23}$ the higher rate of inconclusive SWE results was related mainly to class III obesity (body mass index $\geq 40$ ). Therefore, the CN-CCP identified $8.5 \%$ of our total NAFLD cohort potentially at risk for advanced fibrosis who required a liver specialist referral, and averted hepatology referral for more than $90 \%$ of patients with NAFLD who would otherwise have required assessment by hepatology before the pathway was implemented.

Few studies have reported on the prevalence of NAFLDrelated elevated liver stiffness in the general population. ${ }^{24-27}$ Caballería and colleagues ${ }^{26}$ found that $5.8 \%$ of a Spanish population had liver stiffness, as determined by a transient elastography result greater than $8.0 \mathrm{kPa}$; the majority of patients had NAFLD. In contrast, Koehler and colleagues ${ }^{24}$ reported a prevalence of liver stiffness of $8.4 \%$ with the same cut-off value in a Dutch population. However, in both studies, transient elastography was used to measure liver stiffness for estimating liver fibrosis.

Transient elastography, and SWE and ultrasonography are modalities commonly used to assess liver fibrosis. ${ }^{3}$ These techniques are well validated in patients with NAFLD..$^{15,17,28,29}$

Table 3: Independent predictors of shear wave elastography result of $\mathbf{8} \mathbf{k P a}$ or greater and need for hepatology referral ${ }^{\star}$

\begin{tabular}{|c|c|c|c|c|}
\hline \multirow[b]{2}{*}{ Characteristic } & \multicolumn{2}{|c|}{$\begin{array}{l}\text { Predictors of SWE value } \geq 8 \mathrm{kPa} \\
\text { OR }(95 \% \mathrm{Cl})\end{array}$} & \multicolumn{2}{|c|}{$\begin{array}{l}\text { Predictors of inconclusive result or SWE value } \\
\qquad \geq 8 \mathrm{kPa} \text {, OR }(95 \% \mathrm{Cl})\end{array}$} \\
\hline & Univariate analysis & Multivariate model & Univariate analysis & Multivariate model \\
\hline Age $>60 \mathrm{yr}$ & 2.05 (1.26-3.34) & $1.33(0.75-2.37)$ & $1.49(1.08-2.05)$ & $0.88(0.60-1.31)$ \\
\hline Female sex & $1.68(1.00-2.80)$ & $1.40(0.80-2.48)$ & $1.28(0.93-1.77)$ & $1.14(0.79-1.64)$ \\
\hline $\begin{array}{l}\text { Elevated ALT or AST level at } \\
\text { baseline }\end{array}$ & $1.61(0.97-2.68)$ & NS & $0.96(0.70-1.32)$ & NS \\
\hline Body mass index $\geq 30$ & $2.11(1.10-4.04)$ & $1.93(1.01-3.75)$ & $3.19(2.02-5.06)$ & $2.94(1.85-4.69)$ \\
\hline Diabetes mellitus type 2 & $3.42(2.09-5.60)$ & 2.22 (1.13-4.36) & $3.10(2.24-4.27)$ & 2.33 (1.50-3.63) \\
\hline Impaired fasting glucose & $0.42(0.22-0.79)$ & $0.75(0.33-1.67)$ & $0.52(0.35-0.76)$ & $0.91(0.55-1.49)$ \\
\hline Hypertension & $2.36(1.43-3.89)$ & $2.18(1.19-3.98)$ & $2.61(1.88-3.62)$ & $2.38(1.62-3.50)$ \\
\hline
\end{tabular}


A recent meta-analysis by Hermann and colleagues ${ }^{17}$ showed that, in 156 patients with NAFLD, with the use of liver biopsy as the gold standard, SWE performance was equivalent to that of transient elastography, with a small gain in area under the receiver operating characteristic curve for all stages of fibrosis.

We chose an SWE cut-off value of $8.0 \mathrm{kPa}$, as this cut-off has previously shown excellent performance for ruling out advanced liver fibrosis (sensitivity 91\%, negative predictive value $>95 \%) .{ }^{13,15,17}$ Therefore, we used this SWE cut-off to identify patients with NAFLD at low risk for advanced liver fibrosis, allowing the pathway to direct further focused investigation in patients at risk, recognizing that an SWE cut-off of $8.0 \mathrm{kPa}$ alone has a positive predictive value greater than $75 \%$ for identifying patients with NAFLD with advanced fibrosis (this may be lower in a low-prevalence community setting). ${ }^{13}$ In our cohort, obesity, type 2 diabetes and hypertension were the only independent predictors for an $\mathrm{SWE}$ value of $8.0 \mathrm{kPa}$ or greater. These findings suggest that stratification of patients with NAFLD for risk of advanced liver fibrosis may benefit from targeting patients with these comorbidities.

Almost one-third (31.6\%) of our patients had an FIB-4 index score greater than 1.30 , previously shown to have a negative predictive value of more than $90 \%$ for ruling out advanced fibrosis. ${ }^{13}$ Choosing higher cut-off values for the FIB-4 index score would lead to a higher positive predictive value but lower negative predictive value. ${ }^{13,30,31}$ This is similar to the proportion of patients with NAFLD in a British primary care setting who had an FIB-4 index score greater than $1.30,30 \% .{ }^{32}$ Although not the focus of this paper, a costutility analysis could be conducted comparing SWE results to noninvasive serum fibrosis scores to risk stratify patients with NAFLD.

Shear wave elastography technology is readily applied to most commonly used ultrasonography machines. Our SWEdriven NAFLD pathway decreased primary care referral of patients with NAFLD at low risk to hepatology by more than $90 \%$. This type of triaging of patients with NAFLD within primary care, before specialist referral, will be critical for dealing with the huge and growing burden of patients with NAFLD, allowing streamlined specialist referral for patients at risk for advanced liver fibrosis who may benefit from aggressive intervention targeted at their liver disease.

Furthermore, our study provides data on performance of diagnostic tools for assessing NAFLD. Recent guidelines urged that studies like ours be performed to evaluate the feasibility of routine evaluation for NAFLD in primary care, 3,5 especially with the expected availability of pharmacologic treatment options for patients with NAFLD with advanced fibrosis in the near future.

\section{Limitations}

To allow for easier implementation of the NAFLD pathway, we provided guidance to primary care physicians without strict criteria or incentives to assess only patients with suspected NAFLD. Therefore, most of our type 2 diabetes cohort had NAFLD, since primary care physicians often referred patients with type 2 diabetes who had previous incidental findings of fatty liver for SWE. Similarly, we could calculate FIB-4 index scores only for about two-thirds of our cohort, as primary care physicians in Calgary historically have been discouraged from routinely ordering AST measurement. However, this did not affect our results, as there were no significant differences between patients with NAFLD with available FIB-4 index scores and those without.

Although this was a large primary care-based cohort, only 67 patients had an SWE value of $8 \mathrm{kPa}$ or greater. Future studies with larger samples would be more precise in identifying predictors of elevated liver stiffness.

Currently, SWE is available only in some urban radiologic centres. Furthermore, appropriate training of radiologists and technologists, and the establishment of a proper reporting system are required before SWE can be implemented on a wider scale. We suggest that each jurisdiction implement a pathway to risk stratify patients with NAFLD by means of serum-based or radiologic modalities, or both, based on locally available resources.

Our study aim was to evaluate patients with NAFLD for risk of advanced fibrosis at a specific point in time. Future longitudinal studies are warranted to evaluate patients with NAFLD prospectively and report disease progression among patients at low risk.

\section{Conclusion}

We show the feasibility of implementing a primary carebased clinical care pathway using SWE that facilitates streamlining of specialist referral of patients with NAFLD at risk for advanced liver fibrosis. In our cohort, $8.5 \%$ of patients with NAFLD had elevated liver stiffness or inconclusive results and were referred to hepatology. Using a noninvasive measure like the FIB-4 index would lead to higher rates of specialist referral of patients with NAFLD at low risk for advanced liver fibrosis. Our findings can be used to improve clinical care approaches for evaluation of liver disease in patients with NAFLD and for directing care of patients with NAFLD based on fibrosis risk.

\section{References}

1. Younossi ZM, Koenig AB, Abdelatif D, et al. Global epidemiology of nonalcoholic fatty liver disease: meta-analytic assessment of prevalence, incidence, and outcomes. Hepatology 2016;64:73-84.

2. Ong JP, Younossi ZM. Epidemiology and natural history of NAFLD and NASH. Clin Liver Dis 2007;11:1-16, vii.

3. Chalasani N, Younossi Z, Lavine JE, et al. The diagnosis and management of nonalcoholic fatty liver disease: practice guidance from the American Association for the Study of Liver Diseases. Hepatology 2018;67:328-57.

4. Hagström H, Nasr P, Ekstedt M, et al. Fibrosis stage but not NASH predicts mortality and time to development of severe liver disease in biopsy-proven NAFLD. 7 Hepatol 2017;67:1265-73.

5. Bugianesi E. EASL-EASD-EASO Clinical Practice Guidelines for the management of non-alcoholic fatty liver disease: Disease mongering or call to action? Diabetologia 2016;59:1145-7.

6. Vilar-Gomez E, Martinez-Perez Y, Calzadilla-Bertot L, et al. Weight loss through lifestyle modification significantly reduces features of nonalcoholic steatohepatitis. Gastroenterology 2015;149:367-78.e5; quiz e14-5.

7. Musso G, Cassader M, Rosina F, et al. Impact of current treatments on liver disease, glucose metabolism and cardiovascular risk in non-alcoholic fatty liver disease (NAFLD): a systematic review and meta-analysis of randomised trials. Diabetologia 2012;55:885-904.

8. Tapper EB, Saini SD, Sengupta N. Extensive testing or focused testing of patients with elevated liver enzymes. 7 Hepatol 2017;66:313-9. 
9. Tapper EB, Loomba R. Nonalcoholic fatty liver disease, metabolic syndrome, and the fight that will define clinical practice for a generation of hepatologists. Hepatology 2018;67:1657-9.

10. Younossi ZM, Blissett D, Blissett R, et al. The economic and clinical burden of nonalcoholic fatty liver disease in the United States and Europe. Hepatology 2016;64:1577-86

11. Polanco-Briceno S, Glass D, Stuntz M, et al. Awareness of nonalcoholic steatohepatitis and associated practice patterns of primary care physicians and specialists. BMC Res Notes 2016;9:157.

12. Bedossa P, Patel K. Biopsy and noninvasive methods to assess progression of nonalcoholic fatty liver disease. Gastroenterology 2016;150:1811-22.e4.

13. Xiao G, Zhu S, Xiao X, et al. Comparison of laboratory tests, ultrasound, or magnetic resonance elastography to detect fibrosis in patients with nonalcoholic fatty liver disease: a meta-analysis. Hepatology 2017;66:1486-501.

14. Musso G, Gambino R, Cassader M, et al. Meta-analysis: natural history of non-alcoholic fatty liver disease (NAFLD) and diagnostic accuracy of noninvasive tests for liver disease severity. Ann Med 2011;43:617-49.

15. Cassinotto C, Boursier J, de Lédinghen V, et al. Liver stiffness in nonalcoholic fatty liver disease: a comparison of supersonic shear imaging, FibroScan, and ARFI with liver biopsy. Hepatology 2016;63:1817-27.

16. Kaswala DH, Lai M, Afdhal NH. Fibrosis assessment in nonalcoholic fatty liver disease (NAFLD) in 2016. Dig Dis Sci 2016;61:1356-64.

17. Herrmann E, de Lédinghen V, Cassinotto C, et al. Assessment of biopsyproven liver fibrosis by two-dimensional shear wave elastography: an individual patient data-based meta-analysis. Hepatology 2018;67:260-72.

18. Ogrinc G, Davies L, Goodman D, et al. Squire 2.0 (Standards for Quality Improvement Reporting Excellence): revised publication guidelines from a detailed consensus process. Am 7 Crit Care 2015;24:466-73.

19. Kim HC, Nam CM, Jee SH, et al. Normal serum aminotransferase concentration and risk of mortality from liver diseases: prospective cohort study. BMF 2004;328:983.

20. Sigrist RMS, Liau J, Kaffas AE, et al. Ultrasound elastography: review of techniques and clinical applications. Theranostics 2017;7:1303-29.

21. Barr RG, Ferraioli G, Palmeri ML, et al. Elastography assessment of liver fibrosis: Society of Radiologists in Ultrasound Consensus Conference Statement. Ultrasound Q 2016;32:94-107.

22. Dietrich CF, Bamber J, Berzigotti A, et al. EFSUMB guidelines and recommendations on the clinical use of liver ultrasound elastography, update 2017 (long version) [published erratum in Ultraschall Med 2017;38:e48]. Ultraschall Med 2017;38:e16-47.

23. Kim DW, Suh CH, Kim KW, et al. Technical performance of twodimensional shear wave elastography for measuring liver stiffness: a systematic review and meta-analysis. Korean $\mathcal{F}$ Radiol 2019;20:880-93.

24. Koehler EM, Plompen EP, Schouten JN, et al. Presence of diabetes mellitus and steatosis is associated with liver stiffness in a general population: the Rotterdam study. Hepatology 2016;63:138-47.

25. Mahady SE, Macaskill P, Craig JC, et al. Diagnostic accuracy of noninvasive fibrosis scores in a population of individuals with a low prevalence of fibrosis. Clin Gastroenterol Hepatol 2017;15:1453-60.e1.

26. Caballería L, Pera G, Arteaga I, et al. High prevalence of liver fibrosis among European adults with unknown liver disease: a population-based study. Clin Gastroenterol Hepatol 2018;16:1138-45.e5.

27. Petta S, Di Marco V, Pipitone RM, et al. Prevalence and severity of nonalcoholic fatty liver disease by transient elastography: genetic and metabolic risk factors in a general population. Liver Int 2018;38:2060-8.

28. Cassinotto C, Lapuyade B, Mouries A, et al. Non-invasive assessment of liver fibrosis with impulse elastography: comparison of Supersonic Shear Imaging with ARFI and FibroScan®. 7 Hepatol 2014;61:550-7.
29. Yoneda M, Thomas E, Sclair SN, et al. Supersonic Shear Imaging and transient elastography with the XL probe accurately detect fibrosis in overweight or obese patients with chronic liver disease. Clin Gastroenterol Hepatol 2015;13: 1502-9.e5.

30. Chalasani N, Younossi Z, Lavine JE, et al.; American Gastroenterological Association; American Association for the Study of Liver Diseases; American College of Gastroenterology. The diagnosis and management of non-alcoholic fatty liver disease: practice guideline by the American Gastroenterological Association, American Association for the Study of Liver Diseases, and American College of Gastroenterology. Gastroenterology 2012;142:1592-609.

31. Castera L, Friedrich-Rust M, Loomba R. Noninvasive assessment of liver disease in patients with nonalcoholic fatty liver disease. Gastroenterology 2019;156: 1264-81.e4.

32. Srivastava A, Gailer R, Tanwar S, et al. Prospective evaluation of a primary care referral pathway for patients with non-alcoholic fatty liver disease. 7 Hepatol 2019;71:371-8.

Affiliations: Division of Gastroenterology and Hepatology (Shaheen, Riazi, Kaplan, Burak, Swain), Department of Medicine, University of Calgary; EFW Radiology group (Medellin, Bhayana, Park); Alberta Health Services (Jiang, Schaufert); Community Primary Care, Alberta Health Services (Sargious), Calgary, Alta.

Contributors: Abdel Aziz Shaheen, Alexandra Medellin, Wendy Schaufert, Monica Sargious and Mark Swain conceived and designed the study. Abdel Aziz Shaheen, Alexandra Medellin, Deepak Bhayana, Jason Jiang, Roy Park and Mark Swain acquired the data. Abdel Aziz Shaheen, Kelly Burak, Jason Jiang and Mark Swain analyzed the data. Abdel Aziz Shaheen, Kelly Burak, Alexandra Medellin, Deepak Bhayana, Gilaad Kaplan, Kelly Burak and Mark Swain interpreted the data. Abdel Aziz Shaheen, Alexandra Medellin, Deepak Bhayana, Kelly Burak, Gilaad Kaplan and Mark Swain drafted the manuscript. All of the authors revised the manuscript critically for intellectual content, approved the final version to be published and agreed to be accountable for all aspects of the work.

Funding: This work was supported by the Gilead Sciences InvestigatorSponsored Research Grant Program.

Data sharing: Individual participant data that underlie the results reported in this article (text, tables, figures and appendices) will be available after deidentification. Other documents such as study protocol and statistical analysis plan will also be available. Data will be available beginning 3 months and ending 36 months after article publication to investigators whose proposed use of the data has been approved by an independent review committee identified for this purpose. Shared data should be limited for meta-analysis of individual participant data. Proposals may be submitted up to 36 months following article publication. Proposals should be directed to the corresponding author, Abdel Aziz Shaheen.

Acknowledgement: The authors acknowledge the role of Dr. Robert Myers of Gilead Sciences as a collaborator in the study concept development.

Supplemental information: For reviewer comments and the original submission of this manuscript, please see www.cmajopen.ca/content/8/2/ E370/suppl/DC1. 